\title{
Product Lifecycle Management: Sustainability and knowledge management as keys in a complex system of product development
}

\author{
Maria Giovanna Trotta \\ Politecnico di Torino (ITALY) \\ maria.trotta@polito.it
}

Received February 2010

Accepted October 2010

\begin{abstract}
The PLM process is developing methodologies to support sustainable production during the whole product lifecycle. The present research makes a focus on the first phase product development (the design phase) that is significant to set future costs and environmental impacts. This research analyzes important tools that could be used by PLM to formalize knowledge for sustainable new products development in a company. It offers an overview and compares different approaches to share knowledge, to carry out innovation and to reduce CO2 emissions, materials and energy, as well as developing regulation compliance treated as a design requirement from the conceptual phase through the entire product lifecycle.
\end{abstract}

Keywords: PLM, sustainability, environmental compliance, knowledge

\section{Introduction}

Several international efforts have been made and are in progress to develop and promote studies and applications of sustainability in business and the public sector.

Researches activities are dedicated to the possibility of combining innovative approaches with other tools and methodologies in order to allow a cooperative management to process knowledge in extended value chains, improving efficiency and efficacy. 
Knowledge also can have different levels of complexity based on the relationships and connections with aspects of the IT system. Knowledge can be expressed in different ways as explicit or tacit, the former is technical (objective, known), while the latter is more subjective (linked to specific experiences) and thus difficult to formalize (Van Handenhoven \& Trassaert, 1999).

Increasingly value accrues intellectual experience, which can be acquired, interpreted and transformed to create innovation.

PLM is a recently acquired tool; in itself it represents an innovation to formalizing knowledge, providing a range of different tools for problem solving through ICT.

Product Lifecycle Management is an approach to manage knowledge that involves all phases of product development from concept to disposal.

In the present research the focus is on the first phase of the product development process, where the designer decisions heavily impact on product performances as well as on economic and environmental aspects.

This Paper will offer an overview about tools that can be integrated with the PLM to consider previously and proactively economic, social and environmental impact to start creating a competitive strategy.

\section{Global interests and sustainable development}

The WBCSD (World Business Council for Sustainable Development) is providing a platform for companies to explore sustainable development possibilities, to share knowledge, experiences and best practices and to advocate business positions on these issues in a variety of forums, working with governments, non-governmental and intergovernmental organizations. Members are drawn from more than 30 countries and 20 major industrial sectors. The Council also benefits of a global network of 60 national and regional business councils and regional partners.

The United Nations Environmental Program (UNEP) has called for a "Global Green New Deal" (Barbier \& Edward, 2009) in response to the financial and economic crisis for reviving the global economy, boosting employment and simultaneously accelerating the fight against climate change, environmental degradation and poverty. 
The European Union has developed the ILCD (International Life Cycle Data System) to promote studies and qualitative applications (in line with the international standards on LCA ISO 14040/44) in business and the public sector.

StEP (Solving the E-waste Problem) is an initiative of various UN organizations Together with members from industry, governments, international organizations, NGOs and the science sector actively participating in StEP for the overall aim to solve the e-waste problem and facilitate approaches towards the sustainable handling of e-waste (from electrical and electronic equipment).

\section{PLM approaches for knowledge management}

Via a digital platform for product development, companies can establish a secure computing environment, whereby all subjects involved in the work flow can contribute to the product and process innovation. PLM enables comparison, evaluation and optimization of the different product requirements, linking production information (specifications, models, results of performance, best practices, and reviews) to design. Small and Medium Enterprises have specific needs and limited resources, in these companies integrated systems are often not centralised in only one tool. Fully integrated PLM solutions are designed to provide what small and medium enterprises need to maximize their strategy, and easily scale to meet their future needs.

\subsection{The centralized interfacing}

Centralized interface is a very important approach to manage complex databases, through which individuals can access at data content in other systems connected through the mediation of central flow management. Internet and the Web have facilitated knowledge access by eliminating the physical limitations, enabling the connection to the database via a web browser. Since the management of each system is independent of the central, there must be some shared rules that allow determining who owns the master of each set of data authoring.

\subsection{The integrated platform}

In this approach, all business applications share a single common database managed by an integrated platform, so there is no need for interfaces and there are no translation problems. Business applications, which are supported by this 
architecture, can operate in a transparent manner with greater flexibility in the creation of associative relationships and adapting to the management of complex structures. The integrated approach is able to design, maintain and produce other data, process and metadata management in the database, monitoring its development. Formalization of tacit knowledge as an instrument of innovation

Today it has become essential for companies to develop tools to acquire new knowledge (both internal and external) to quickly adapt to changes and remain competitive in the market (Nonaka, 1994).

Much of the knowledge within a company belongs to the "front-line workers" especially if they feel an integral part of it. These workers are important figures in the innovation development.

One of the methods used to monitor their knowledge is to extract meaning from text documents that workers have exchanged and then classify it into categories, discovering hidden associations, extracting specific information for the creation of ontology learning and their description by the syntactic parser or systems of knowledge mining (Kaufman \& Michalski, 2005).

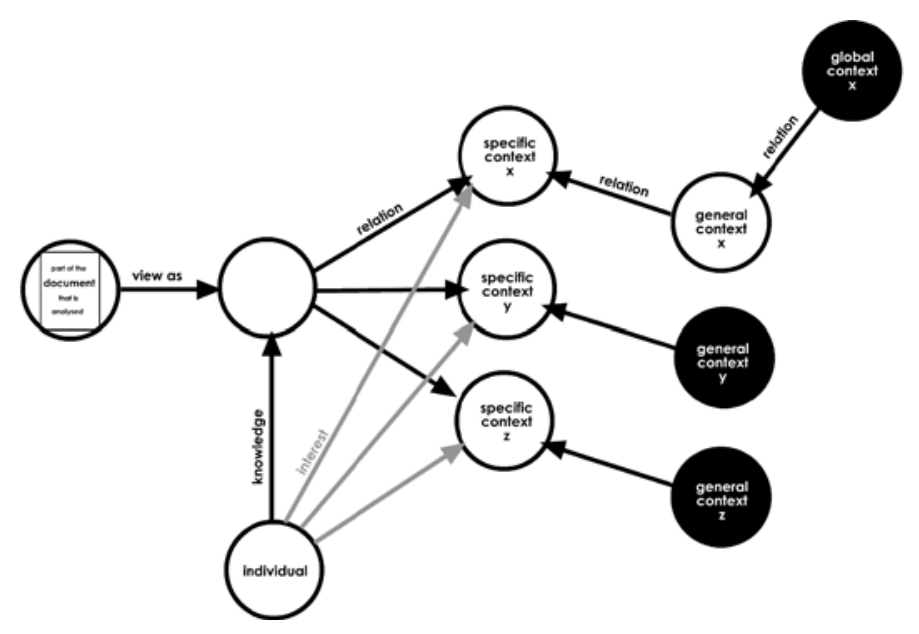

Figure 1. "Example of conceptualization network".

Tacit knowledge is linked by a set of implicit relations that are established between the elements. This is not simple to outline, one of the most appropriate forms for its representation is associative with weighted connections (Rumelhart, McClelland, $\&$ the PDP Research Group, 1986). Such a system is exactly half way between the 
data mining systems and those of knowledge mining. It represents a viable option for the management of so-called intellectual capital businesses.

This type of system is able to update the knowledge or user acknowledging the context in which it operates by analyzing the new type of information. Dialogue, environmental communication and cooperation with customers, consumer groups, partners increase the chances of experience exchanging and useful knowledge to mutual inspiration.

\section{$4 \quad$ Life Cycle thinking}

The depletion of some natural resources, rising commodity prices, environmental regulations that constrain the use of the artefact, in line with the requirements of eco-compliance and the need to continually reduce costs are driving companies to seek new ways to optimize their consumption that can be controlled from the design phase through a PLM environment. Sustainable design is not a one-size-fitsall concept, but must be adapted on the life cycle profile of the specific product, the business strategy, and the culture and capabilities of the organization. Further, it can be applied to both goods and services (Dobbs \& Cormican, 2007).

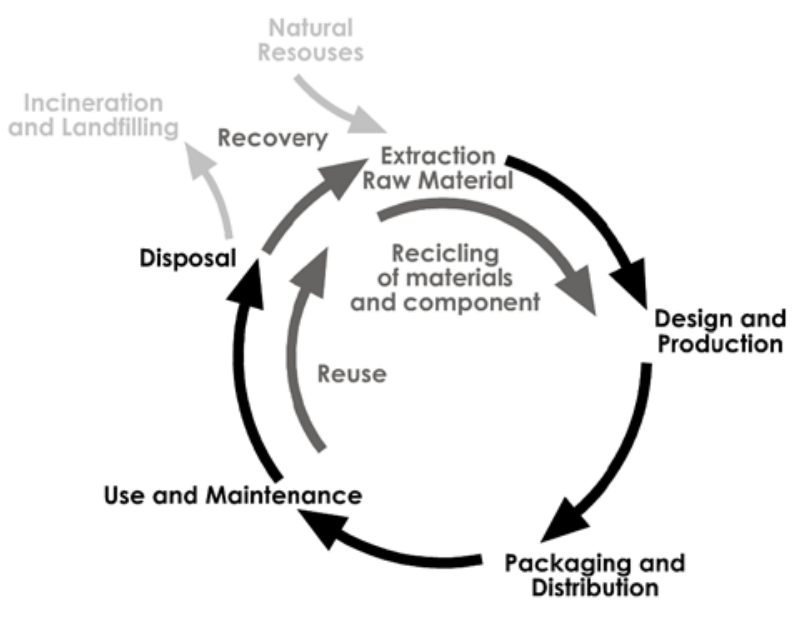

Figure 1. "Life Cycle Thinking". Source: UNEP

\subsection{Life Cycle Assessment (LCA)}

LCA and LCT have developed a scientific methodology that derives from TRACI (methodology studied by EPA to describe the environmental condition in USA). 
LCA is an objective tool accepted at an international level (ISO 14000), it identifies and quantifies in a systematic way the amount of energy and material used and substances released in the environment, during all stages of the life cycle from the raw material extraction until product disposal.

LCA is divided in four main phases: firstly "Goal and scope definition" (ISO 14041)in which the analysis is planned (functional units, data requirements, the geographical area, methodology to assign potential impacts); secondly " Inventory analysis" (ISO 14041) that involves data collection and calculation of inputs and outputs for each process considered; thirdly "Impact Assessment" (ISO 14042) that aims at evaluating for each inventory element the significance in terms of environmental impacts (global warming, acidification, eutrophication, solid waste, land use, ozone depletion, photochemical ozone formation, toxicity for humans, resource use), characterization, normalization and weighting according with ISO; fourthly "Interpretation" (ISO 14043) that is the combination of the results from the previous phases with the conclusions and recommendations consistent with goal and scope can be reached. Much software (eg. Gabi, SimaPro, and Bounsted) support the different types of LCA approaches.

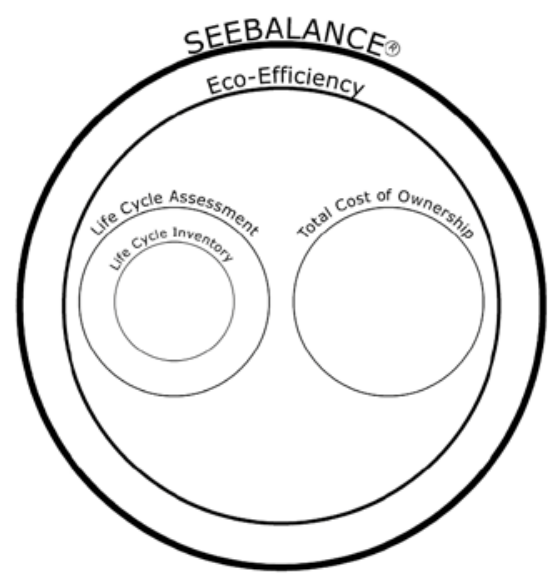

Figure 3. "Difference between Eco Efficiency Analysis and Life Cycle Analysis". Source: BASF et al. (2002-2005)

The PLM process is developing methodologies for the development of green products that seek to provide solutions combining LCA with economic analysis. 


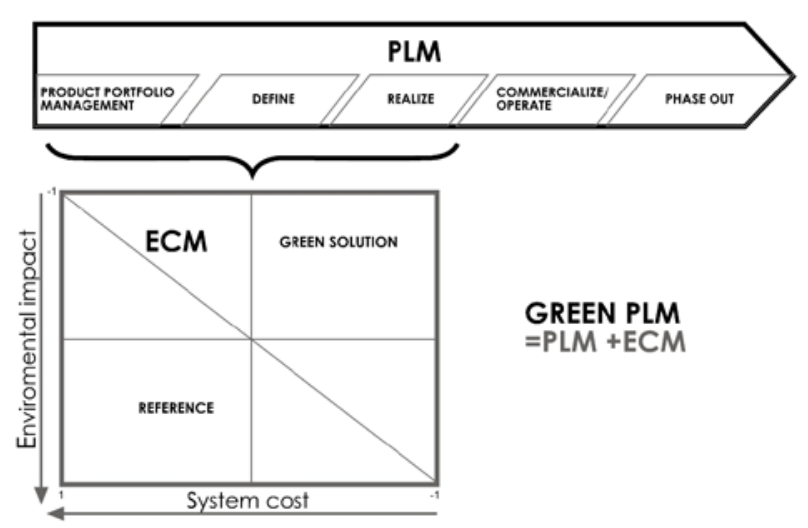

Figure 4. "Eco Care Matrix".

BASF (chemical company) has developed Eco Efficiency Analysis to compare quantitatively processes considering the entire life cycle and environmental and economic aspects, starting from the Life Cycle Assessment and Total Costs.

Siemens has combined the ECM (Eco Care Matrix by BASF) and PLM thereby demonstrating environmental and economic (OPerating EXpense, CAPital EXpenditure) performances (Wegener, Finkbeiner, Geiger, Olsen, \& Walachowicz, 2009).

\subsection{TRIZ}

Theory of Inventive Problem Resolution (TRIZ) provides a logical methodology that follows evolutionary laws and finite principles for inventive approaches.

The methodology was developed in Russia in 1946 by Genrich Altshuller, who analysed more than 200.000 patents to extract technological knowledge and experiences by the best inventive minds that can be used by engineer and designers to develop new ideas in their projects.

Triz considers five levels of inventions according to the fact that problem solutions are in the same industrial membership area.

TRIZ principally consists of a series of: laws of evolution, forty principles (trends of engineering systems), thirty-nine contradictions (technical-engineering and physical).

The TRIZ methodology follows the steps to start considering: 


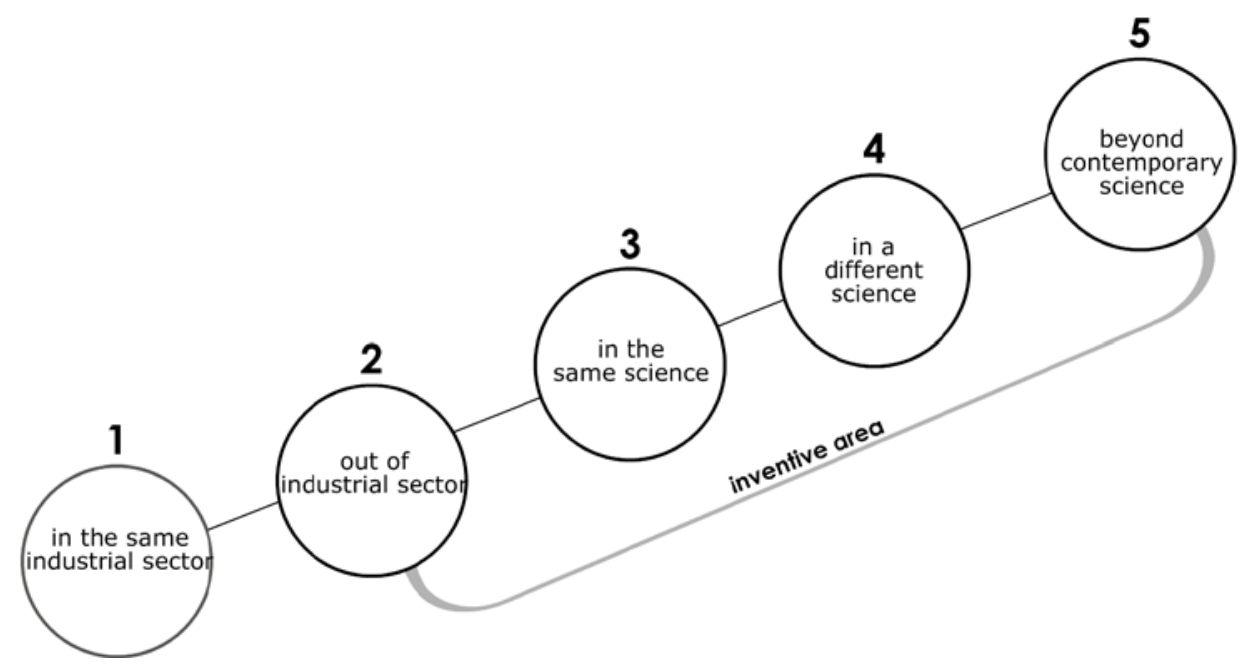

Figure 4. "TRIZ inventive levels".

1. theory and the eight trends of engineering systems;

2. analysis (of Function-related problems, contradictions and new perspectives, match objectives with function lists provided in TRIZ, reinterpretation step by step with the algorithm for inventive problems solving Ariz.);

3. knowledge based tools to validate the solution (tools to suggest transformations as forty inventive Principles, Seventy-six Standard Solutions, the data base for effects of knowledge base);

4. verification of solutions (all the concepts developed will be analyzed to verify the technical and economic viability, assigning a priority value according to technical and productive aspects).

Several researches are dedicated to provide validation of TRIZ as en environmental methodology.

The WBCSD (World Business Council for Sustainable Development) has identified seven eco-principles for developing environmentally-friendly products and reducing their impact:

A. Reduce the material intensity of its goods and services (material reduction).

B. Reduce the energy intensity of its goods and services (energy reduction). 
C. Reduce the dispersion of any toxic materials (toxicity reduction).

D. Enhance the recyclability of its materials (material retrieval).

E. Maximize the sustainable use of renewable resources (resource sustainable).

F. Extend the durability of its products (product durability).

G. Increase the service intensity of its goods and services (product service).

Each element improves simultaneously eco-efficiency of products or services that are produced (Desimone \& Popoff, 1997), through links between each element of eco-efficiency with the parameters of engineering, the reduction in weight, size, quantity of material used. These parameters are correlated with the others related to TRIZ (Liu \& Chen, 2001).

The trends, towards an ideal design, meet all the requirements of eco-efficiency and follow specific parameters of importance hierarchy (AHP Analytic Hierarchy Process) that can be identified through LCA in the design project (Fussler \& James, 1996).

The AHP technique is introduced for convenience in the weighting of abstract objects. AHP matrix is composed of seven elements of eco-efficiency. Subsequently, the designer can use this table to find the corresponding TRIZ engineering parameters. CAD software highlights the capabilities of the design through FEA (finite element analysis) or 3D CAD software. There exist contradictions that require the integration of these with other parameters.

CAD Eco Design tool is designed to provide technical support for developing ecoinnovative products through eco-design targets and evaluating the environmental performance of products, obtaining practical engineering parameters of TRIZ, and through case reference (Chang \& Chen, 2004).

\subsection{Design for environment}

Consumers are becoming more attentive, favouring companies that progress toward environmental compliance and sustainability. 


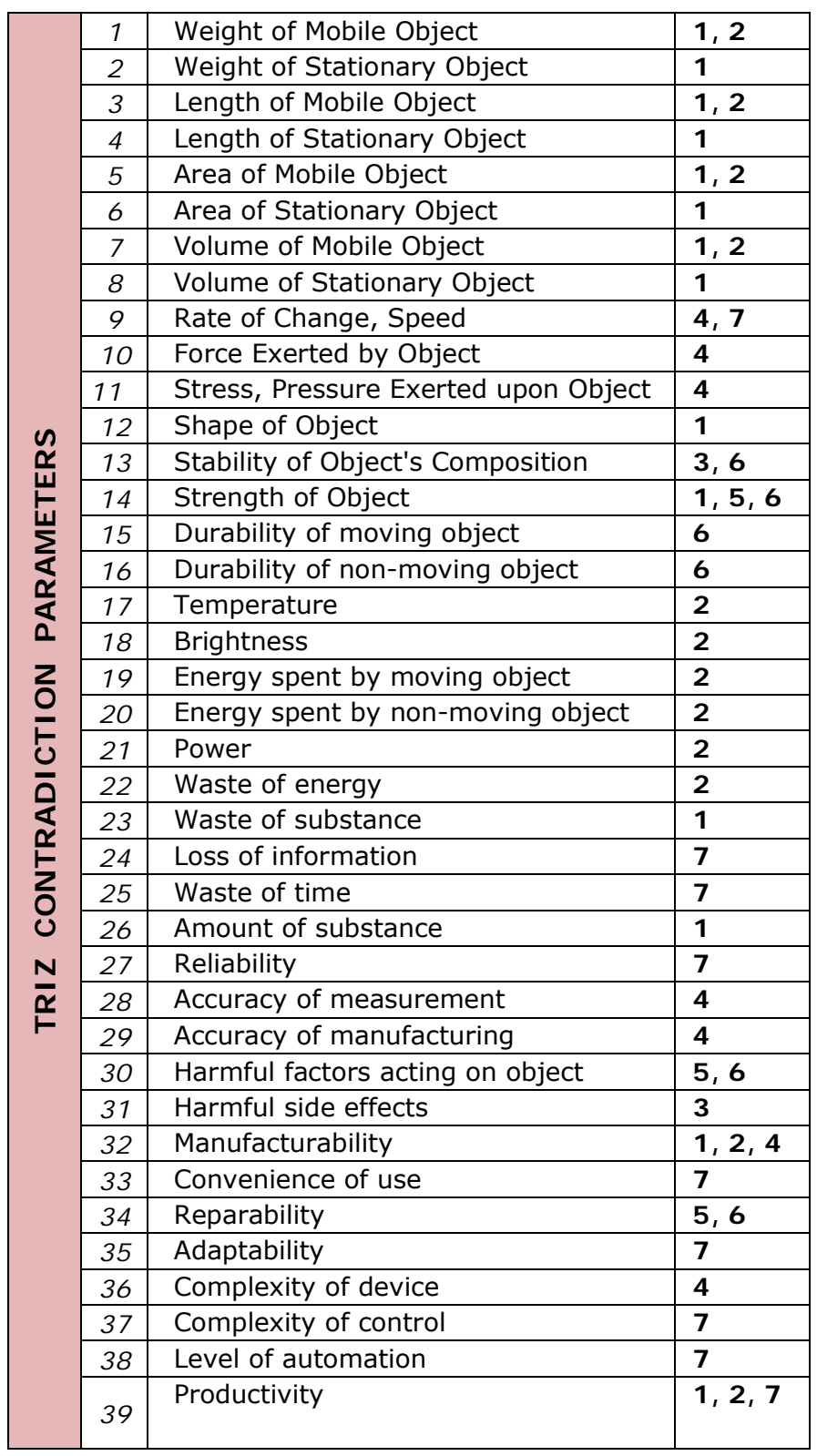

\begin{tabular}{|c|c|c|}
\hline $\mathbf{1}$ & Material intensity & \\
\hline 2 & Energy intensity & م \\
\hline 3 & Toxic material & m \\
\hline 4 & Recyclability & I \\
\hline 5 & Renewable Resources & $=$ \\
\hline 6 & Durability & $\bar{\nabla}$ \\
\hline 7 & Service Intensity & 恧 \\
\hline
\end{tabular}

Table 1. "WBCSD Eco-Principles". Source: Chang \& Chen (2004).

The engineers and designers team creates a preliminary layout of the product, material selection, manufacturing processes and dimensions. In the parametrical part of design process, it's applies Design for $X(D f X)$ tools to refine the choices made in the previous steps. Design for the Environment includes the option of Life Cycle Thinking which considers all the steps of the life cycle right from the design step. The DFE in a simplified condition considers two main levels: the product enhancements and analysis. The various criteria are measured primarily by two software tools: the DFE manual Workbench (charts and information) that is 
integrated and adaptable in a automated CAD environment. It provides an analysis, synthesis and evaluation for the improvements for the entire life cycle with an average level of analysis (Roche, 2004).

The workbench structure consists of five elements:

1. Impact Assessment System which is a simplification of the analysis of the life cycle through the use of a quantification on Eco indicator 95;

2. The Structure Assessment Method SAM collects information about the materials, disassembly time, the connections number, material compatibility and so on;

3. The advisor agent in charge of providing a quantified list of items that are the cause of the high environmental impact of product that concerns the IAS and the SAM, suggesting material choice to reduce it;

4. Knowledge agent that is a tool to search materials with specific performance;

5. The Report Generator allows a comparison with the initial condition.

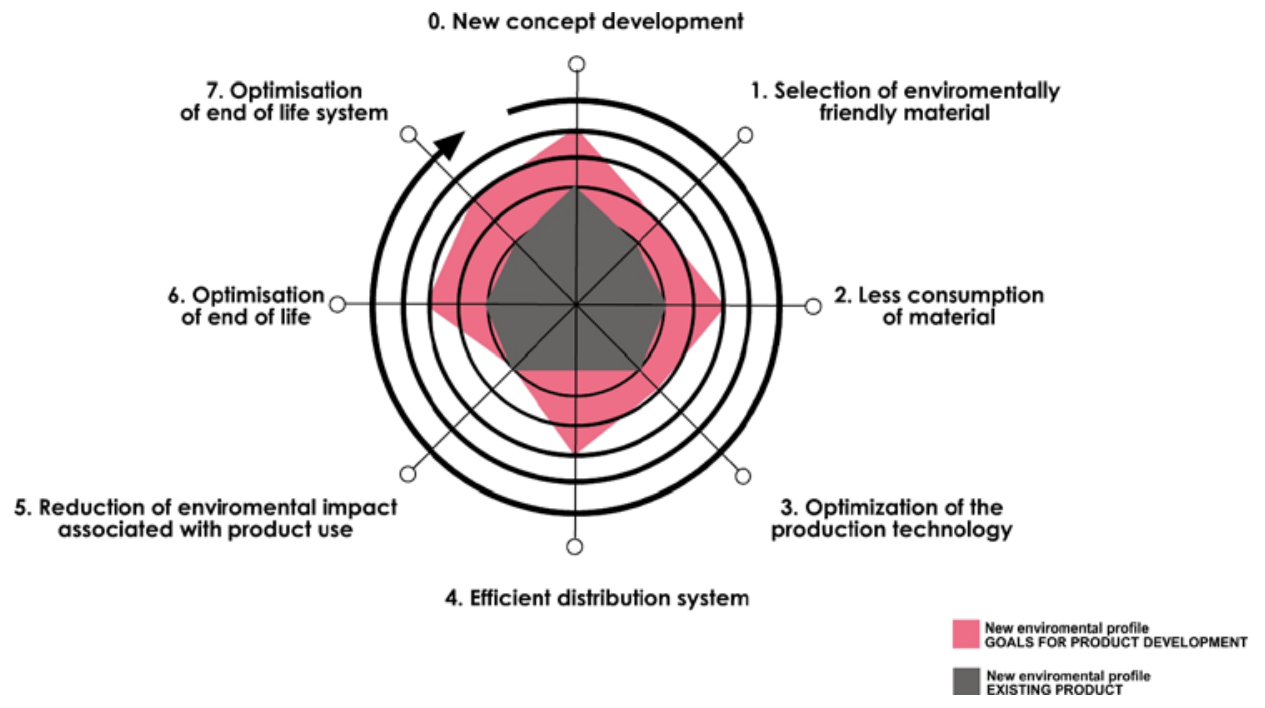

Figure 5. "A product's environmental profile".

Scientific tests were carried out by a large number of experienced engineers using a methodology with analysis, synthesis, evaluation and improvement, with aim to implement approaches for enterprises for rapid roll out to the associated tiers in the relevant sectors. 


\section{Conclusion}

Exchanging information, sharing experiences of experts and continue developing an environmental portfolio, reducing $\mathrm{CO} 2$ emissions, addressing environmental considerations starting from the design phase of new products through correct mapping of the functional units, it's becoming essential in today's dynamic market.

Product design influences the social, economic and environmental spheres. In the first step of production, therefore, companies need to have both the general and specific view of relationships and influences of a new product design into the environment. A virtual processes' overview of the life cycle of the new product can be done through the integration of the information needed to control improvement and consequent changes in relations with each transformation defined level by level.

One way, to design a new eco-friendly product, is to start with non-polluting processes, less energy, resources, waste and making project economically effective.

Environmental regulations, (RoHS, WEEE and REACH) that could be viewed by companies as threats, can become an opportunity to be competitive.

Several researches activities are dedicated to the possibility of combining PLM, DFE, TRIZ, LCA offering approaches, methodologies, tools to support the design teamwork as early as possible to minimise errors, time, costs for improving product quality and facilitate a sustainable environment.

\section{References}

Barbier, E.B. (2009, April). A Global Green New Deal. Retrieved from http://uwacadweb.uwyo.edu/barbier/Publications/A\%20Global\%20Green\%20New \%20Deal-Executive\%20Summery.pdf

BASF SE, Institute for Geography and Geo-ecology of Karlsruhe University, Ökoinstitut e.V., \& Jena University (2002-2005). Sustainable Aromatics Chemistry' research project. Retrieved from www.basf.com/group/corporate/en/sustainability/eco-efficiency-analysis/index 
Chang, H.T., \& Chen, J.L. (2004). The conflict-problem-solving CAD software integrating. TRIZ into eco-innovation. Advances in engineering software, 35, 8-9. doi:10.1016/j.advengsoft.2004.06.003

DeSimone, L.D., \& Popoff, F. (1997). Eco-efficiency: the business link to sustainable development. Cambridge: Massachusetts Institute of Technology.

Dobbs, J., \& Cormican, K. (2007, July). A design for environment product analysis. Paper presented at the 4th International on Product Life Cycle Management Conference, Kilometro Rosso, Italy.

Fussler, C. \& James, P. (1996). Driving Eco-Innovation. London: Pitman Publishing.

IES Institute for Environment and Sustainability. (2009). ILCD supporting tools. Retrieved from http://Ict.jrc.ec.europa.eu/assessment/tools

Kaufman, K., \& Michalski, R.S. (2005). From Data Mining to Knowledge Mining. In Rao, C.R., Solka, J.L. \& Wegman, E.J. (Eds.), Handbook in Statistics vol. 24 (pp. 47-75). North Holland: Elsevier.

Liu, C.C., \& Chen, J.L. (2001, December). Development of product green innovation design method. Paper presented at the Second International Symposium on Environmentally Conscious Design and Inverse Manufacturing, Tokyo, Japan.

Nonaka, I. (1994). A Dynamic Theory of Organizational Knowledge Creation. Organization Science, 1(5), 14-. doi:10.1287/orsc.5.1.14

Roche, T. (2004). The Design for environmental compliance workbench tool. In D. Talab \& T. Roche (Eds), Product Engineering (pp. 3-16). Netherlands: Springer.

Rumelhart, D.E., McClelland, J.L., \& the PDP research group. (1986). Explorations in the microstructure of cognition. Cambridge: MIT Press.

Van Handenhoven, E., \& Trassaert, P. (1999). Design knowledge and design skills. Paper presented at International Conference on Engineering Design (ICED 99), Munich.

Wegener, D., Finkbeiner, M., Geiger, D., Olsen, S.I., \& Walachowicz, Y. (2009, September). Comprehensive Approach to Energy and Environment in the Eco 


\section{Care Program for Design. Paper presented at RISO International Energy Conference, Copenhagen.}

(C) Journal of Industrial Engineering and Management, 2010 (www.jiem.org)

\section{(c)}

Article's contents are provided on a Attribution-Non Commercial 3.0 Creative commons license. Readers are allowed to copy, distribute and communicate article's contents, provided the author's and Journal of Industrial Engineering and Management's names are included. It must not be used for commercial purposes. To see the complete license contents, please visit http://creativecommons.org/licenses/by-nc/3.0/. 